ARTICLE

Received 23 Aug 2014 | Accepted 13 Aug 2015 | Published 13 Oct $2015 \quad$ DOl: 10.1038/ncomms9369

\title{
Tamoxifen augments the innate immune function of neutrophils through modulation of intracellular ceramide
}

Ross Corriden 1,2, Andrew Hollands', Joshua Olson', Jaclyn Derieux²,3, Justine Lopez ${ }^{4}$, John T. Chang ${ }^{4}$, David J. Gonzalez ${ }^{2,3} \&$ Victor Nizet ${ }^{1,3,5}$

Tamoxifen is a selective oestrogen receptor modulator widely used for the treatment of breast cancer. In addition to its activity as an oestrogen receptor agonist/antagonist, tamoxifen also modulates sphingolipid biosynthesis, which has been shown to play an important role in the regulation of neutrophil activity. Here, we find that tamoxifen stimulation enhances several pro-inflammatory pathways in human neutrophils, including chemotaxis, phagocytosis and neutrophil extracellular trap (NET) formation. The enhancement of NET production occurs via

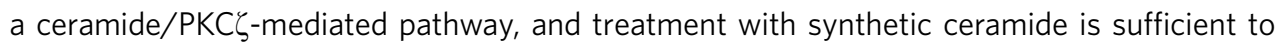
promote NET formation. Pretreatment of human neutrophils with tamoxifen boosts neutrophil bactericidal capacity against a variety of pathogens in vitro and enhances clearance of the leading human pathogen methicillin-resistant Staphylococcus aureus in vivo. Our results suggest that tamoxifen, and the lipid signalling pathways it modulates, merit further exploration as targets for boosting host innate immune function.

\footnotetext{
${ }^{1}$ Department of Pediatrics, University of California, San Diego, La Jolla, California 92093, USA. ${ }^{2}$ Department of Pharmacology, University of California, San Diego, La Jolla, California 92093, USA. ${ }^{3}$ Skaggs School of Pharmacy and Pharmaceutical Sciences, University of California, San Diego, La Jolla, California 92093, USA. ${ }^{4}$ Department of Medicine, University of California, San Diego, La Jolla, California 92093, USA. ${ }^{5}$ Rady Children's Hospital, San Diego, California 92123, USA. Correspondence and requests for materials should be addressed to R.C. (email: rcorriden@ucsd.edu) or to V.N. (email: vnizet@ucsd.edu).
} 
T amoxifen, a selective oestrogen receptor modulator taken by hundreds of thousands of patients for the treatment of breast cancer $^{1}$, is included on the World Health Organization's list of essential medicines due to its cost effectiveness and safety profile ${ }^{2}$. In addition to its activity as an oestrogen receptor agonist/antagonist ${ }^{3}$, tamoxifen has many other effects that include modulation of sphingolipid biosynthesis ${ }^{4}$. Such 'off-target' effects have been shown to contribute to the therapeutic action of tamoxifen ${ }^{5}$; however, its impact on some normally functioning cell types, in particular circulating neutrophils, remains poorly understood. Earlier investigations of the impact of tamoxifen on neutrophil function have largely been limited to its inhibition of transendothelial migration, suggesting a primary immunosuppressant activity 6,7 . Because sphingolipids are known to be important modulators of immune cell function ${ }^{8}$, we investigated the effects of tamoxifen on human neutrophils, the primary phagocytic leukocyte of the immune system.

Using in vitro and in vivo approaches, we find that tamoxifen, in addition to enhancing neutrophil chemotaxis and phagocytosis, strongly promotes the formation of DNA-based neutrophil extracellular traps (NETs). NETs, which are coated with antimicrobial peptides, histones and/or granule proteases, are capable of entrapping and killing bacteria and other pathogens ${ }^{9,10}$. Tamoxifen enhances killing of pathogens in vitro and enhances clearance of methicillin-resistant Staphylococcus aureus (MRSA) in vivo. We find that such alterations in neutrophil activity are dependent on intracellular ceramide.

\section{Results}

Direct assessment of $N$-Formyl-Met-Leu-Phe (fMLP)-induced neutrophil chemotaxis indicated that tamoxifen, while not stimulating chemotaxis by itself (Supplementary Fig. 1), significantly enhanced directional cell migration in response to fMLP stimulation (Fig. 1a). These differences in findings may be due to variations in protocol (for example, previous studies pre-incubated cells with tamoxifen and removed it before assessment of chemotaxis rather than assessing migration in its presence) or the added complexity of in vitro transendothelial migration models. In addition to these findings, fluorescencebased quantification of extracellular DNA release revealed that tamoxifen (Fig. 1b), its active metabolite 4-hydroxytamoxifen (Fig. 1b) and its primary metabolite $N$-desmethyltamoxifen (Supplementary Fig. 2), strongly stimulated NET production in freshly isolated human neutrophils. These results were confirmed by immunostaining of stimulated neutrophils using a primary antibody against myeloperoxidase, a NET marker (Fig. 1c) ${ }^{11}$. Because tamoxifen is generally described to have antagonist activity at the oestrogen nuclear receptors $\operatorname{ER} \alpha$ and $\operatorname{ER} \beta$ and agonist activity at the G-protein-coupled oestrogen receptor GPR30, we attempted to replicate these results using selective agonists/antagonists. In these studies, NET production was not observed in response to treatment with Methyl-piperidinopyrazole (MPP) or pyrazolo [1,5-a] pyrimidine (PHTPP) (selective $\mathrm{ER} \alpha$ and ER $\beta$ antagonists, respectively ${ }^{12,13}$ ), G-1 (a GPR30 agonist ${ }^{14}$ ) or fulvestrant (a selective oestrogen receptor modulator that mimics the mixed agonist/antagonist characteristics of tamoxifen ${ }^{15,16}$ ) (Supplementary Fig. 3), indicating that tamoxifen-induced NET production is mediated by an oestrogen receptor-independent mechanism.

To elucidate the pathway underlying the neutrophil-activating effects of tamoxifen, we next assessed signalling molecules/ effectors typically associated with NET release. Although NET production has been reported to be dependent on reactive oxygen species (ROS) generation ${ }^{17}$, we found that both tamoxifen and 4-hydroxytamoxifen lowered ROS production in human neutrophils (Fig. 1d), mirroring our previous findings with statins $^{18}$. This finding was supported by experiments using the ROS scavenger butylated hydroxyanisole, which potently inhibited phorbol myristate acetate (PMA)-induced NET production, but did not significantly reduce tamoxifen-induced NET production (Fig. 1e).

\section{C}

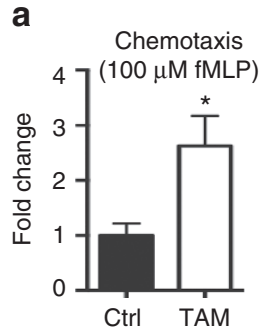

b
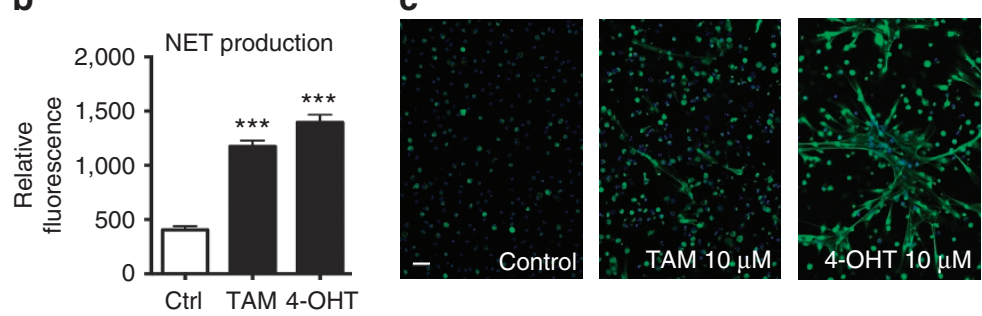

e

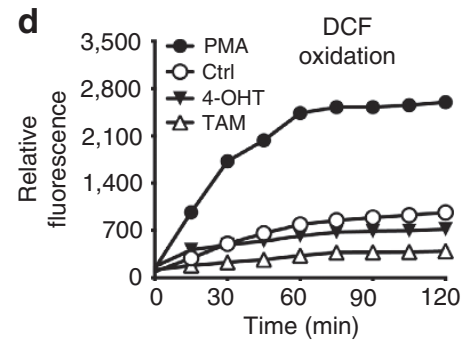

Figure 1 | Tamoxifen promotes extracellular trap formation in human neutrophils in vitro. (a) The effect of $10 \mu \mathrm{M}$ tamoxifen (TAM; upper well) on chemotaxis in the presence or absence of $100 \mathrm{nM}$ fMLP (lower well) was determined using a transwell system $(n=12)$. (b) Extracellular DNA was quantified to determine the effect of both TAM and 4-hydroxytamoxifen $(4-\mathrm{OHT})$ on NET release $(n=9)$. (c) NET production was confirmed by fixing and immunostaining TAM-/4-OHT-stimulated cells with DAPI and a primary antibody for myeloperoxidase (blue: DAPI; green: NETs/myeloperoxidase); images representative of three independent experiments shown (scale bar $=50 \mu \mathrm{m}$ ). (d) DCF-based ROS assays were performed to evaluate the effect of TAM and 4-HT on ROS production $(n=9)$. (e) Neutrophils were pre-incubated with the ROS scavenger BHA before addition of either PMA to TAM to determine whether ROS production is required for tamoxifen-induced NET production $(n=9)$. Where applicable, results were analysed by one-way analysis of variance and post hoc Newman Keuls test. ${ }^{\star} P<0.05$, ${ }^{\star \star \star} P<0.001$ versus control values. BHA, butylated hydroxyanisole; DAPI, 4',6-diamidino-2phenylindole; NS, not significant; ROS, reactive oxygen species. 

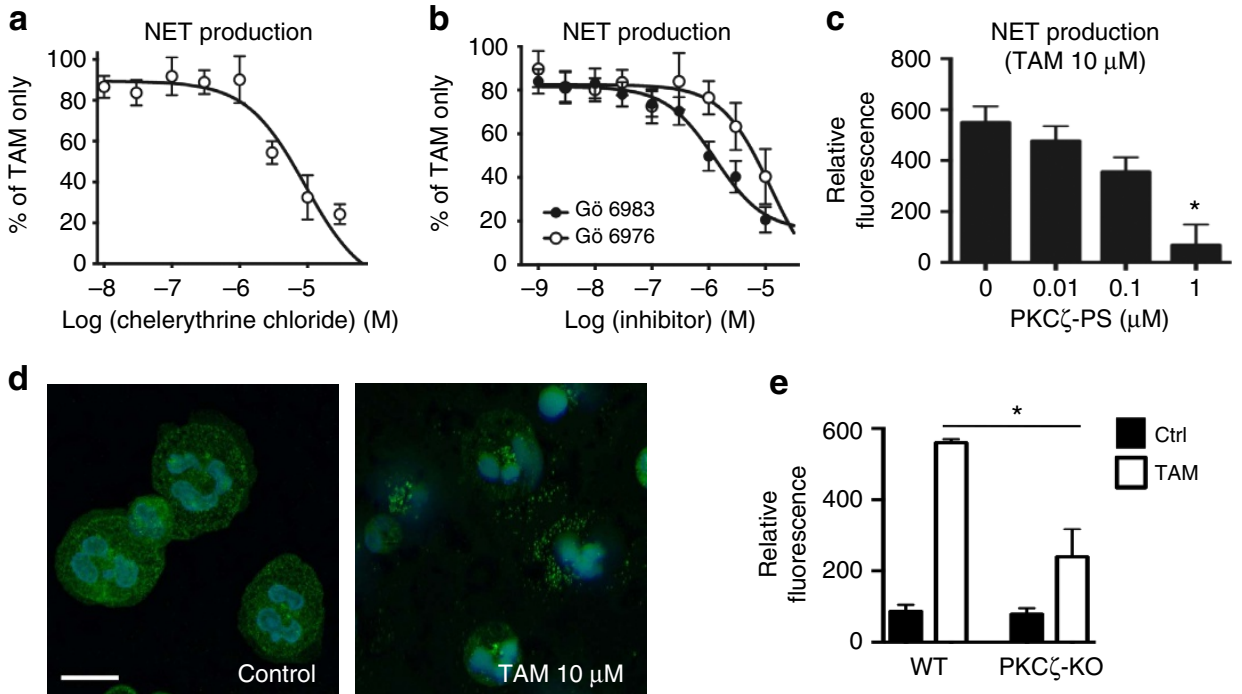

Figure 2 | Tamoxifen-induced extracellular trap production is PKC $\zeta$ dependent. (a) To determine whether PKC is required for TAM-induced NET production, neutrophils were pre-incubated with (a) the general PKC antagonist chelerythrine chloride $(n=8)$ or $(\mathbf{b})$ the more selective inhibitors Gö 6976

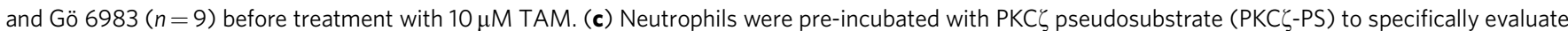

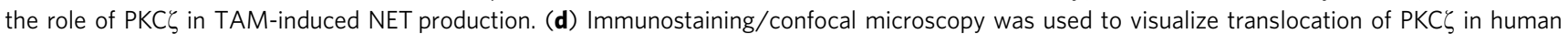
neutrophils in response to TAM $(10 \mu \mathrm{M})$ treatment (blue: 4',6-diamidino-2-phenylindole; green: PKC $)$; images representative of three independent experiments are shown (scale bar, $10 \mu \mathrm{m}$ ). (e) Bone marrow-derived neutrophils were collected from PKC $\zeta$ knockout mice to determine TAM-induced NET production in comparison with wild-type (WT) littermate controls $(n=4-5)$. Where applicable, results were analysed by one-way analysis of variance and post hoc Newman Keuls test. ${ }^{\star} P<0.05$ versus control values.

In contrast, pre-incubation with the general PKC antagonist chelerythrine chloride inhibited tamoxifen-induced NET production (Fig. 2a). To determine the specific PKC isoform responsible for tamoxifen-induced NET release, we stimulated neutrophils with tamoxifen in the presence of two broadspectrum PKC inhibitors with different selectivity profiles, Gö 6973 and Gö 6983, the latter of which is a more potent inhibitor of PKC $\zeta$ and PKC $\mu$ (ref. 19). Inhibition curves revealed that Gö 6983 exhibited greater inhibition of tamoxifen-induced NET production, pointing to either $\mathrm{PKC \zeta}$ or $\mathrm{PKC} \mu$ as the primary PKC isoform responsible for this induction (Fig. 2b). Further experiments using a highly selective inhibitor of $\mathrm{PKC}$, $\mathrm{PKC} \zeta$ pseudosubstrate $(\mathrm{PKC} \zeta-\mathrm{PS})^{20}$, resulted in near-complete inhibition of tamoxifen-induced NET production, strongly implicating $\mathrm{PKC} \zeta$ as a key mediator of this process (Fig. 2c). Inhibition of PI3K had no effect on tamoxifen-induced NET production (Supplementary Fig. 4), although tamoxifen promoted translocation of neutrophil PKCל (Fig. 2d). The importance of $\mathrm{PKC} \zeta$ in tamoxifen-induced NET production was confirmed in experiments using bone marrow-derived neutrophils collected

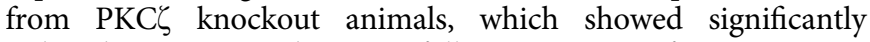
reduced NET production following tamoxifen treatment compared with wild-type littermate controls (Fig. 2e).

Through its ability to inhibit glucosylceramide synthase, a key enzyme in the sphingolipid synthesis pathway that converts ceramide to glucosylceramide, tamoxifen has been shown in some cell types to promote intracellular accumulation of ceramide, a potent PKC $\zeta$ activator $^{21}$. Thus, we next determined whether ceramide accumulation could drive tamoxifen-induced NET production. Indeed, similar to tamoxifen, ceramide promoted NET release in a concentration-dependent manner (Fig. 3a,b) while inhibiting ROS generation (Fig. 3c). Ceramide-induced NET production was also $\mathrm{PKC} / \mathrm{PKC} \zeta$ dependent (Fig. 3d). Mass spectrometry revealed a statistically significant increase in C16 and C24 ceramide species in neutrophils upon tamoxifen treatment. Structural verification of ceramide species was obtained with high mass accuracy of the parent ion and additionally by monitoring the hallmark 264-Dalton ion fragment released during the fragmentation of ceramides via tandem mass spectrometry (Fig. 3e; Supplementary Fig. 5). Elevated intracellular ceramides were also visually confirmed via immunocytochemistry using an anti-ceramide antibody (Fig. 3f). Further evidence of the role of ceramide in NET production was obtained by treating cells with D,L-threo-1-phenyl-2decanoylamino-3-morpholino-1-propanol (DL-PDMP), a selective inhibitor of glucosylceramide synthase that mimics the intracellular ceramide-increasing effect of tamoxifen ${ }^{22}$, which also promoted NET production (Fig. 3g). Sphingosine-1-phosphate, which is generated from ceramide via the cascade initiated by glucosylceramide synthase ${ }^{23}$, did not significantly promote NET production (Supplementary Fig. 6). To further confirm that tamoxifen-induced NET production is ceramide dependent, we pre-incubated neutrophils with myriocin, an inhibitor of serine palmitoyltransferase ${ }^{24}$. Inhibition of this enzyme, which initiates the pathway that generates ceramide from palmitoylCoA, prevents tamoxifen-mediated ceramide accumulation ${ }^{25}$. Although it does not directly inhibit PKCל (Supplementary Fig. 7), myriocin treatment resulted in complete inhibition of tamoxifen-induced NET production (Fig. $3 \mathrm{~h}$ ) and partially reversed inhibition of PMA-induced ROS production by tamoxifen (Supplementary Fig. 8), illustrating the key role of ceramide accumulation in this pathway.

Having established that tamoxifen stimulates neutrophil migration and induces NET production, we examined whether neutrophil bactericidal capacity could be enhanced via tamoxifen treatment. At the concentrations used to stimulate neutrophils, tamoxifen and 4-hydroxytamoxifen had negligible effects on the growth of the USA300 strain of community-associated MRSA, Escherichia coli (K1 serotype) or Pseudomonas aeruginosa (PA01 strain) (Fig. 4a). However, both tamoxifen and 4-hydroxytamoxifen enhanced the killing activity of activated human neutrophils against unopsonized bacteria of all three species (Fig. 4b). Treatment of neutrophils with ceramide mimicked these effects (Supplementary Fig. 9). Live/dead staining 


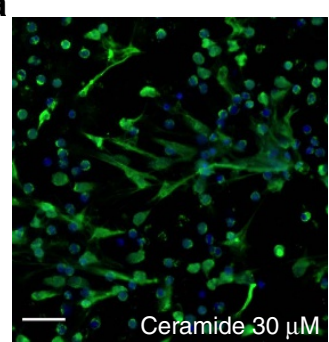

d

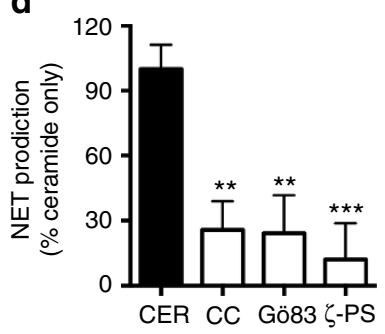

9

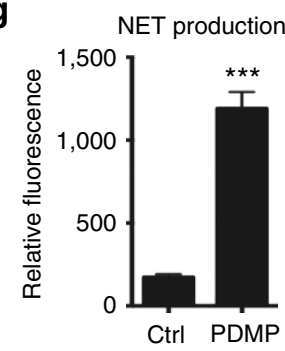

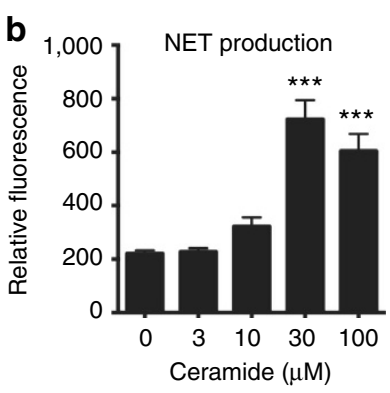

e
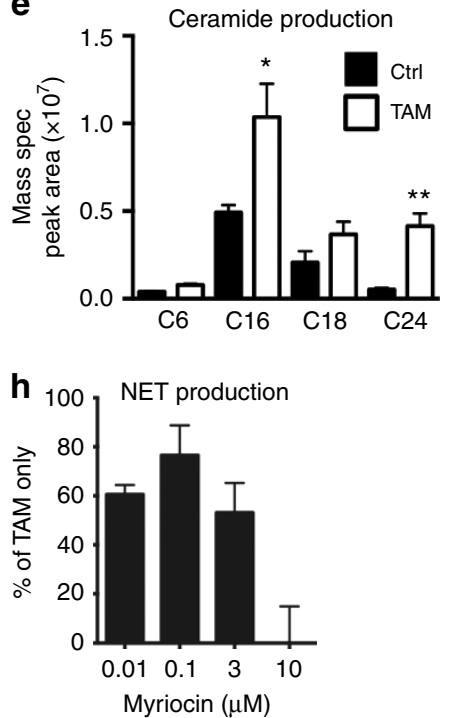

C

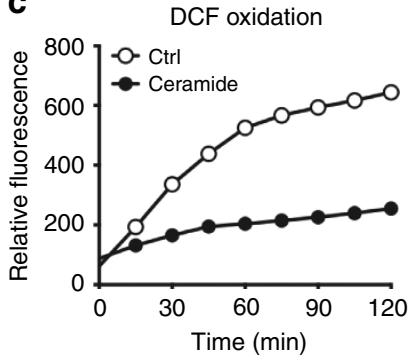

f

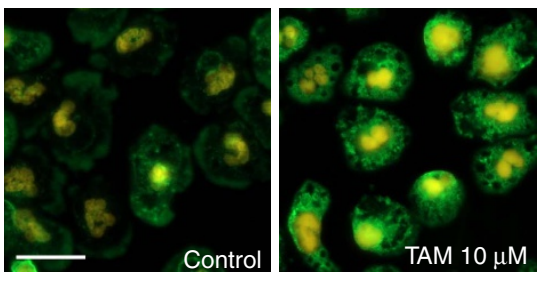

i

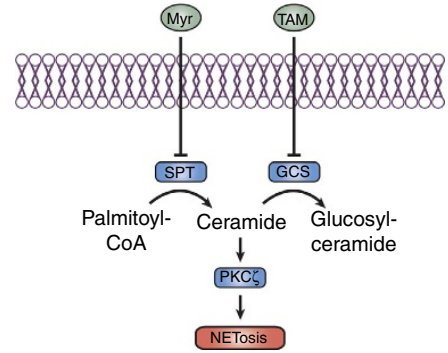

Figure 3 | Tamoxifen-induced NET production is mediated by intracellular ceramide. (a) Ceramide-induced NET production was visualized by immunostaining neutrophils for myeloperoxidase (blue: DAPI; green: NETs/myeloperoxidase); images representative of three independent experiments are shown (scale bar, $50 \mu \mathrm{m})$. (b) Quantification of extracellular DNA/NET production in response to ceramide treatment $(n=9)$. (c) The effect of ceramide $(30 \mu \mathrm{M})$ on ROS production was determined using DCF-based ROS assays $(n=9)$. (d) Neutrophils were pre-incubated with PKC inhibitors (CC = chelerythrine chloride, Gö83 = Gö 6983, $\zeta$-PS $=$ PKC $\zeta$ pseudosubstrate; $10 \mu \mathrm{M}$ each; $n=9$ ) to determine the involvement of PKC in ceramideinduced NET production. (e) Mass spectrometry analysis of lipid extracts was performed to quantify intracellular accumulation of several ceramide species in response to TAM treatment $(10 \mu \mathrm{M})$. (f) Intracellular ceramide levels in control and TAM-treated $(10 \mu \mathrm{M})$ neutrophils were visualized via immunostaining of fixed neutrophils using a ceramide primary antibody (green: ceramide); images representative of three independent experiments are shown, with identical exposure and gain settlings used for control and TAM-treated samples (scale bar, $20 \mu \mathrm{m}$ ). (g) The effect of DL-PDMP, which similar to tamoxifen inhibits glucosylceramide synthase, on NET production was assessed via quantification of extracellular DNA ( $n=9$ ). (h) Myriocin, which blocks ceramide synthesis by inhibiting serine palmitoyltransferase, concentration dependently inhibits tamoxifen-induced NET production. (i) Summary of the mechanism of tamoxifen-induced NET production. By inhibiting glucosylceramide synthase, tamoxifen treatment leads to an accumulation of neutrophil intracellular ceramide levels, which promotes NET formation via activation of PKC $\zeta$. Where applicable, results were analysed by one-way analysis of variance and post hoc Newman Keuls test. ${ }^{\star} P<0.05,{ }^{\star \star} P<0.01,{ }^{\star \star \star} P<0.001$ versus control values. ROS, reactive oxygen species.

of USA300 MRSA exposed to either control or tamoxifen-treated neutrophils revealed dead bacteria within the NETs of stimulated neutrophils, in contrast to mostly live bacteria surrounding the untreated neutrophils producing fewer NETs (Fig. 4c). Addition of DNAse to neutrophils before the addition of bacteria significantly enhanced bacterial recovery (Fig. 4d), suggesting that the bactericidal effects of tamoxifen are largely NET driven. Addition of DNase to neutrophil/bacteria culture samples before serial dilution and plating indicated that reductions in colonyforming units (CFUs) were primarily due to killing, rather than clumping, of bacteria (Supplementary Fig. 10). Several different approaches confirmed that the DNAse used in these experiments was active, being able to both degrade DNA and eliminate NETs (Supplementary Fig. 11). Given that some reports have suggested that the principal activity of NETs is to clump rather than kill bacteria ${ }^{26,27}$, our results provide evidence that NETs are heterogeneous in both their mechanism of formation and activity. Both tamoxifen and 4-hydroxytamoxifen also stimulated the uptake of S. aureus-labelled bioparticles (Supplementary Fig. 12), suggesting a further activity to upregulate phagocytic pathways. Additional experiments using USA300 MRSA opsonized by incubation in pooled human serum also revealed enhanced bactericidal activity of neutrophils following a 10-min preincubation with tamoxifen (Fig. 4e).

We next tested tamoxifen in a murine model of systemic MRSA infection. At $1 \mathrm{~h}$ following intraperitoneal (i.p.) treatment with tamoxifen or vehicle control (corn oil), outbred CD-1 mice were infected i.p. with a lethal dose of USA300 MRSA. Mice were subsequently treated with tamoxifen or vehicle control at 1 and $8 \mathrm{~h}$ post infection. Tamoxifen provided significant protection against mortality (Fig. 5a); whereas all mice in the control group died on day $1,40 \%$ of the tamoxifen-treated mice remained alive at day 3 and $33 \%$ surviving to the experimental end point (5 days). In subsequent experiments, mice were treated with tamoxifen or vehicle $2 \mathrm{~h}$ before and $6 \mathrm{~h}$ after i.p. infection with an enhanced green fluorescent protein (EGFP)-expressing USA300 
a

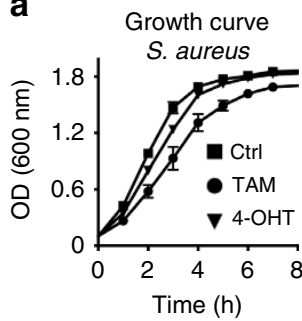

b
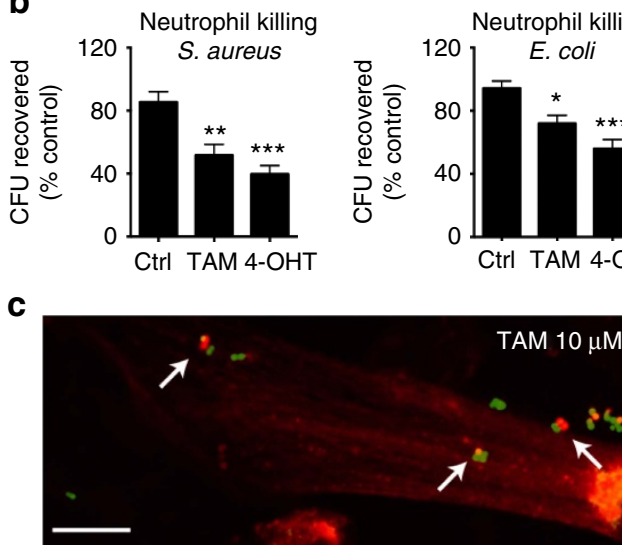

Growth curve

E. coli
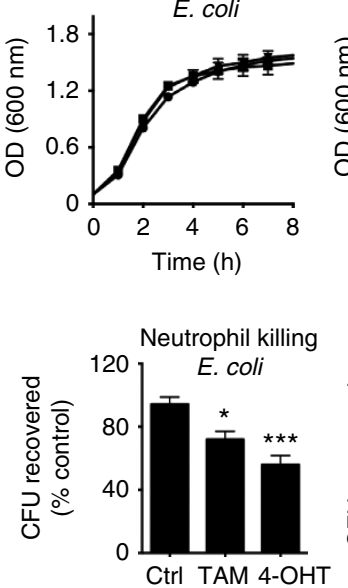

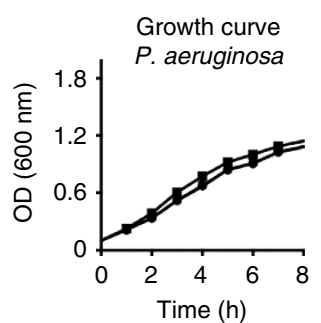

Neutrophil killing d Neutrophil killing

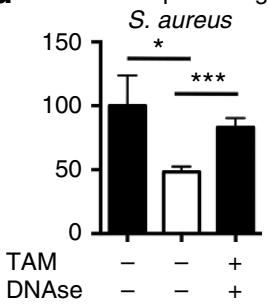

e

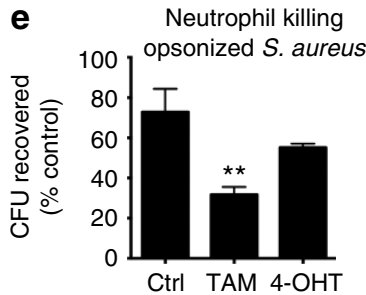

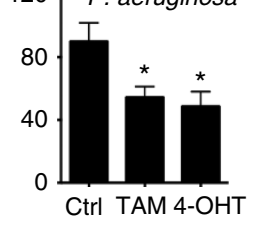

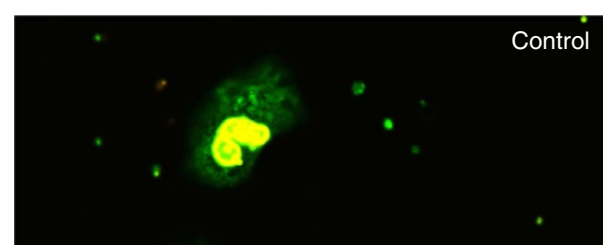

Figure 4 | Tamoxifen enhances neutrophil killing. (a) Bacterial growth curves for USA300 methicillin-resistant Staphylococcus aureus (MRSA), K1 strain E. coli or PA01 strain P. aeruginosa $(n=6)$ in the presence or absence of $10 \mu \mathrm{M}$ TAM or 4-hydroxytamoxifen (4-OHT). (b) Bactericidal assays evaluating the effect of a 4-h TAM or 4-OHT pre-treatment on neutrophil killing (represented by a reduction of CFUs) of USA300 MRSA, E. coli and P. aeruginosa $(n=9-12)$. (c) Live/dead cell analysis of fixed cells; dead bacteria (red: propidium iodide, indicated by arrows) can be observed in tamoxifen-stimulated NETs (left panel). Live bacteria (green: SYTO 8) can be observed outside of the NETs and surrounding unstimulated cells (right panel). Images shown are representative of three independent experiments (scale bar, $10 \mu \mathrm{m}$ ). (d) Addition of DNAse before addition of bacteria to tamoxifen-treated neutrophils significantly reduces killing $(n=9)$. (e) Neutrophils were incubated with TAM and bacteria concurrently to determine killing of USA300 MRSA opsonized using pooled human serum $(n=8-9)$. Where applicable, results were analysed by one-way analysis of variance and post hoc Newman Keuls test. ${ }^{\star} P<0.05$, ${ }^{\star \star} P<0.01,{ }^{\star \star \star} P<0.001$ versus control values.

MRSA. Mice were killed $8 \mathrm{~h}$ post infection to assess bacterial dissemination; mice in the tamoxifen-treated group exhibited significantly lower bacterial counts in peritoneal lavage fluid as well as in the liver, kidney, spleen and blood (Fig. 5b), with bacterial counts below the limit of detection in the tissues of several tamoxifen-treated animals. Analysis of peritoneal lavage fluid using fluorescence microscopy revealed very few intact bacteria in samples from tamoxifen-treated mice, in stark contrast to samples collected from vehicle-treated animals, whose neutrophils contained large numbers of intact bacteria (Fig. $5 c, d$ ). Of note, neutrophils from these control animals often appeared damaged, consistent with previous findings that $S$. aureus can promote neutrophil necrosis following phagocytosis ${ }^{28}$.

\section{Discussion}

In sum, we have shown that tamoxifen enhances neutrophil bactericidal activity, with increased NET production a likely contributory mechanism. Our evidence suggests that this enhancement occurs in significant part through the modulation of intracellular ceramide levels. Ceramide, an important bioactive sphingolipid and cellular stress signal, has previously been linked to neutrophil apoptosis ${ }^{29,30}$, and tamoxifen-induced ceramide accumulation mediates certain oestrogen receptor-independent effects of the drug against cancer tissues ${ }^{5}$. Here, we show that both tamoxifen and synthetic ceramide are potent inducers of NETosis, a novel cell death pathway with an important role in innate immunity ${ }^{31}$. Interestingly, ceramide can activate both apoptotic and autophagocytic pathways ${ }^{32}$, the latter of which have recently been implicated in NET production ${ }^{33}$. Shifting the balance between apoptotic and autophagocytic pathways may influence relative levels of NET production; indeed, we found that rapamycin, a potent inducer of autophagy, enhanced ceramide-induced NET production while having little effect on its own (Supplementary Fig. 13).

Further investigation of compounds that modulate the ceramide biosynthesis pathway may reveal more potent enhancers of neutrophil antimicrobial activity. Along these lines, it is interesting to note that the active metabolite of tamoxifen, 4-hydroxytamoxifen, is even more potent at enhancing NET release and bactericidal activity than tamoxifen. Exploring the mechanism underlying tamoxifen/4-hydroxytamoxifen-induced NET formation may facilitate discovery of derivative compounds specifically optimized to promote targeted enhancement of innate immune responses.

The outcomes of enhanced NET production are likely to be pathogen specific. Several bacterial species are capable of evading NET capture through various mechanisms (for example, via the expression of extracellular nucleases capable of cleaving DNA-based structures ${ }^{34,35}$ ). In the context of S. aureus, a focus of this study, tamoxifen may exhibit particularly marked effects due to the pathogen's ability not only to evade killing via opsonophagocytosis ${ }^{36,37}$ and oxidative burst ${ }^{38}$, but also to invade neutrophils and trigger a unique necrolytic pathway. We observed neutrophils in peritoneal lavage samples collected from tamoxifen-treated mice exhibited a significantly reduced bacterial load in comparison with untreated controls.

In addition to their role in innate immunity, excessive or aberrant production of NETs has been implicated in inflammatory diseases such as vasculitis and bronchial asthma ${ }^{39,40}$. The 

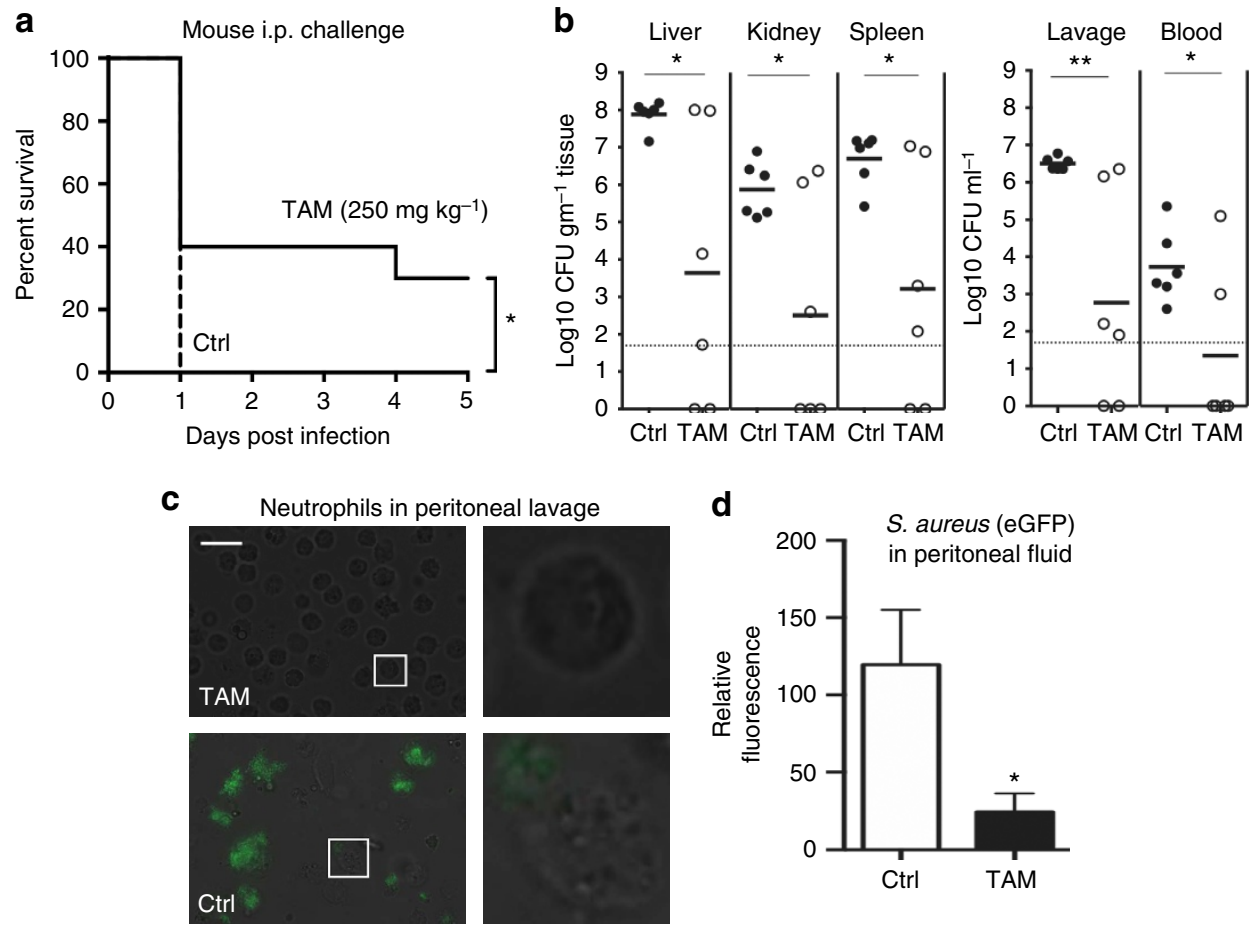

Figure 5 | Tamoxifen reduces mortality and enhances clearance of USA300 MRSA in an in vivo model of acute infection. (a) At $1 \mathrm{~h}$ following intraperitoneal (i.p.) treatment with tamoxifen (TAM; $250 \mathrm{mg} \mathrm{kg}^{-1}$ ) or vehicle control (VEH; corn oil), CD-1 background mice were infected i.p. with a lethal dose of USA300 MRSA $\left(5 \times 10^{8} \mathrm{CFU} \mathrm{ml}^{-1}, 200 \mu \mathrm{l}\right)$. Mice were subsequently treated with tamoxifen or vehicle control at 1 and $8 \mathrm{~h}$ post infection and monitored for 5 days. Tamoxifen significantly protected against mortality. (b) To assess bacterial dissemination, CD-1 background mice received i.p. injections of tamoxifen $\left(250 \mathrm{mg} \mathrm{kg}^{-1}\right.$ ) or vehicle control $2 \mathrm{~h}$ before and $6 \mathrm{~h}$ following i.p. injection of enhanced green fluorescent protein (EGFP)-expressing USA300 MRSA $\left(5 \times 10^{8} \mathrm{CFU} \mathrm{ml}^{-1}, 200 \mu \mathrm{l}\right)$. Bacterial counts were determined in the peritoneal lavage fluid and blood, kidney, liver and spleen tissue samples. (c) Light microscopy revealed that neutrophils in the vehicle control samples often appeared damaged and contained multiple intact bacteria (scale bar, $25 \mu \mathrm{m}$ ). (d) Quantitative analysis of fixed peritoneal lavage samples confirmed the presence of elevated EGFP-USA300 MRSA levels in vehicleversus tamoxifen-treated animals. Results were analysed by the log-rank test (survival curve comparison) or one-way analysis of variance and post hoc Newman Keuls test. ${ }^{\star} P<0.05,{ }^{\star \star} P<0.01$ versus control values.

cumulative effect of tamoxifen on neutrophil responses hold clinical implications, given the large number of patients receiving this drug and related therapeutic agents.

\section{Methods}

Materials. Tamoxifen citrate, ceramide, MPP dihydrochloride, G-1, Gö 6983, Gö 6976, PKCל-PS and DL-PDMP were all obtained from Tocris Bioscience (Bristol, UK). Myriocin was obtained from Cayman Chemical (Ann Arbor, MI). Unless otherwise noted, all other chemicals were obtained from Sigma Aldrich, St Louis, MO.

Bacterial strains. MRSA strain USA300 (UAMS 1182), E. coli strain K1 and $P$. aeruginosa strain PA01 were used in this study. EGFP-transformed MRSA strain USA300 (LAC) were used for in vivo studies where indicated.

Neutrophil isolation. Human venous blood was collected from healthy volunteers, with heparin added as an anticoagulant. Neutrophils were isolated using Polymorphprep (Axis-Shield, Dundee, Scotland) according to the manufacturer's protocol. Blood was drawn according to a protocol approved by the local ethics committee. Neutrophils were collected from PKC $\zeta$ knockout animals (C57BL/6 background) and wild-type littermate controls by extracting bone marrow from harvested tibia. Bone marrow was plated on polystyrene dishes and incubated for $37^{\circ} \mathrm{C}$ with $5 \% \mathrm{CO}_{2}$ for $1 \mathrm{~h}$; subsequently, neutrophils (still in suspension) were collected, counted and used in experiments.

Transwell chemotaxis assay. Neutrophils, pre-incubated for $10 \mathrm{~min}$ at $24^{\circ} \mathrm{C}$ in Hanks' Balanced Salt Solution (HBSS) alone or HBSS with $10 \mu \mathrm{M}$ tamoxifen, were seeded in 6-mm transwell permeable supports ( $3 \mu \mathrm{m}$ pore size; Corning Inc.) that were placed in 24-well plates; lower chambers contained either HBSS alone, or $100 \mathrm{nM}$ fMLP. Following a 45 -min incubation at $37^{\circ} \mathrm{C}$, inserts were removed and cells were lysed by addition of Triton X-100 ( $0.1 \%$ final, $\left.10 \mathrm{~min}, 24^{\circ} \mathrm{C}\right)$. To determine the relative levels of migration to the lower well, the colorimetric elastase substrate $N$-methoxysuccinyl-Ala-Ala-Pro-Val-p-nitroanilide was added to the lysed cell samples (10 mM final); following a 30 -min incubation at $24^{\circ} \mathrm{C}$, absorbance at $405 \mathrm{~nm}$ was measured using a SpectraMAX Gemini EM fluorescence reader (Molecular Devices, Sunnyvale, CA).

Induction and quantification of NETs. To induce extracellular trap production, neutrophils were seeded in 96-well plates at a density of $2 \times 10^{5}$ cells per well in HBSS (final volume: $200 \mu \mathrm{l}$ ). Where applicable, cells were incubated with NETosis inhibitors at $37^{\circ} \mathrm{C}$ with $5 \% \mathrm{CO}_{2}$ for $60 \mathrm{~min}$ (myriocin), $45 \mathrm{~min}$ (PKC $30 \mathrm{~min}$ (all other inhibitors) before addition of NET-inducing agents. NET inducers were then added, and cells were incubated for a further $2 \mathrm{~h}$ at $37^{\circ} \mathrm{C}$ with $5 \% \mathrm{CO}_{2}$. Where applicable, $25 \mathrm{nM}$ PMA was included as a positive control. Micrococcal nuclease was then added (final concentration: $500 \mathrm{mU} \mathrm{ml}^{-1}$ ) before an additional 10 -min incubation at $37^{\circ} \mathrm{C}$. Micrococcal nuclease activity was stopped via the addition of $5 \mathrm{mM}$ EDTA, and plates were spun at $200 \mathrm{~g}$ for $8 \mathrm{~min}$. Supernatant samples $(100 \mu \mathrm{l})$ were collected from each well, and extracellular DNA content was quantified using a Quant-IT PicoGreen dsDNA Assay kit (Life Technologies, Carlsbad, CA) according to the manufacturer's instructions.

NET visualization and fluorescence microscopy. To visualize NETs, cells were seeded on 24-well plates at a density of $2 \times 10^{5}$ cells per well. Following stimulation of NET production $\left(2 \mathrm{~h}, 37^{\circ} \mathrm{C}\right.$ with $5 \% \mathrm{CO}_{2}$ with indicated drugs), cells were fixed by the addition of paraformaldehyde ( $4 \%$ final) for $10 \mathrm{~min}$ at $24^{\circ} \mathrm{C}$. After blocking for 45 min with PBS containing $2 \%$ bovine serum albumin (2\% PBS-BSA) and $2 \%$ goat serum, cells were immunostained via the following incubation steps (each at room temperature): $1 \mathrm{~h}$ with rabbit anti-human myeloperoxidase primary antibody (1:300 in 2\% PBS-BSA; Dako North America, Inc., Carpinteria, CA; cat \# A039829-2), $45 \mathrm{~min}$ (protected from light) with Alexa Fluor 488 goat anti-rabbit immunoglobulin G secondary antibody (1:500 in 2\% PBS-BSA); Life Technologies, cat \# A11070) and $10 \mathrm{~min}$ with $1 \mu \mathrm{M}$ Hoechst-33342-trihydrochloride. Three washes with PBS were performed after each staining step. Images were obtained using a Zeiss AxioObserver D1 microscope equipped with an LD A-Plan $\times 20 / 0.35$ $\mathrm{Ph} 1$ objective. Imaging of intracellular ceramide was performed by permeabilizing 
cells with $0.25 \%$ Triton X-100 following fixation with paraformaldehyde, blocking with $2 \%$ PBS-BSA and $2 \%$ donkey serum for $1 \mathrm{~h}$, followed by incubation for $1 \mathrm{~h}$ with mouse anti-ceramide primary antibody (1:300 in 2\% PBS-BSA; Sigma Aldrich; cat \# C8104-50TST) and $45 \mathrm{~min}$ (protected from light) with Alexa Fluor 488 donkey anti-mouse immunoglobulin G secondary antibody (1:500 in 2\% PBS-BSA; Life Technologies; cat \# A21202). Representative images shown were collected the Zeiss AxioObserver D1 microscope and objective described above, with exposure and gain settings kept consistent during collection of control and tamoxifen-treated images. A similar protocol in combination with a PKCל primary antibody (1:200 in 2\% PBS-BSA; Abcam, Cambridge, MA, cat \# ab59364) was used to visualize intracellular PKC $\zeta$ distribution using a Leica TCS SPE confocal microscope equipped with a HC PLAN APO $\times 63 / 1.40-0.60$ numerical aperture oil immersion objective. For live/dead cell analysis, neutrophils were incubated in eight-well LabTek II glass-bottom slides (Nalge Nunc, Penfield, NY) for $2 \mathrm{~h}$ with or without tamoxifen, followed by addition of USA300 MRSA (multiplicity of infection (MOI): 5). After $30 \mathrm{~min}$, cells were stained using a LIVE/DEAD BacLight Bacterial Viability kit according to the manufacturer's instructions. Cells were fixed with $4 \%$ paraformaldehyde and imaged using the Leica TCS SPE confocal microscope and objective described above.

ROS production assays. Neutrophils were incubated in HBSS containing $10 \mu \mathrm{M}$ $2^{\prime}, 7^{\prime}$-dichlorofluorescein diacetate for $20 \mathrm{~min}$ at $37^{\circ} \mathrm{C}$ with gentle agitation. Neutrophils were then centrifuged at $400 \mathrm{~g}$ for $5 \mathrm{~min}$, washed with HBSS and centrifuged again using the same settings before being counted and resuspended in HBSS at a concentration of $5 \times 10^{6}$ cells per ml. Samples of the cell suspension were then added to a 96-well plate $\left(5 \times 10^{5}\right.$ cells per well $)$ and mixed at a 1:1 ratio with PMA, ceramide, tamoxifen or 4-hydroxytamoxifen. Fluorescence intensity ( $485 \mathrm{~nm}$ excitation, $530 \mathrm{~nm}$ emission) was measured in 15 -min intervals using a SpectraMAX Gemini EM fluorescence reader over the course of $2 \mathrm{~h}$ (plates were incubated at $37^{\circ} \mathrm{C}$, protected from light and with gentle shaking, between reads).

Extraction and quantification of ceramide species. Cellular lipids were extracted from neutrophils using the protocol described by Zhang et al. ${ }^{41}$ Samples obtained from these extractions were analysed using Orbitrap Fusion Tribrid mass spectrometry technology. Briefly, nano ultra-HPLC was performed on a Thermo Fisher EASY-nLC (Thermo Fisher Scientific Inc., Waltham, MA) with an incorporated cooled autosampler compartment calibrated for a $6 \times 8$-sample grid. The flow rate was set to $300 \mathrm{nl} \mathrm{min}^{-1}$, and an 85 -min gradient was run. Mobile phase A consisted of $0.1 \%$ formic acid in water and mobile phase B consisted of $0.1 \%$ formic acid in acetonitrile. The nano-analytical column was prepared by pulling Polymicro fused silica, $30 \mathrm{~cm} \times 75 \mu \mathrm{m}$, (Polymicro Technologies, Phoenix, AZ) and packing with $3 \mu \mathrm{m}$ UChrom C18 bulk-packing material (nanoLCMS Solutions LLC). Analysis was performed on a finely calibrated Thermo Fisher Orbitrap Fusion Tribrid mass spectrometer (Thermo Fisher Scientific Inc.). Positive electrospray ion mode was used in all runs in the targeted selective ion-monitoring scan mode. Relative quantification of the structurally confirmed ceramide species was determined by integration of the peak areas obtained from the extracted ion chromatogram using Xcalibur software.

Bacterial growth curves. Glass tubes containing Todd Hewitt broth plus vehicle (10\% HBSS), tamoxifen $(10 \mu \mathrm{M})$ or 4-hydroxytamoxifen $(10 \mu \mathrm{M})$ were inoculated with sufficient amounts of overnight bacterial cultures to achieve an optical density $(600 \mathrm{~nm})$ of 0.1 . Tubes were incubated in a shaking $37^{\circ} \mathrm{C}$ incubator, and absorbance measurements at $600 \mathrm{~nm}$ were taken every hour up to $7 \mathrm{~h}$, and once at $24 \mathrm{~h}$, using a Spectronic 20D + spectrophotometer (Thermo Scientific, Waltham, MA, USA).

In vitro bactericidal assays. Freshly isolated neutrophils in serum-free RPMI were added to 48 -well plates at a density of $4 \times 10^{5}$ cells per well. HBSS, tamoxifen or 4-hydroxytamoxifen was added to applicable wells, resulting in a final volume of $200 \mu \mathrm{l}$, and cells were incubated for $4 \mathrm{~h}$ at $37^{\circ} \mathrm{C}$ with $5 \% \mathrm{CO}_{2}$ (identical wells containing no neutrophils were also prepared). Overnight cultures of bacteria were resuspended in RPMI containing $10 \% 70^{\circ} \mathrm{C}$ heat-inactivated fetal bovine serum to achieve a density of $8 \times 10^{5}$ CFUs per ml. Fifty microlitre of this bacterial suspension was added to applicable wells of the 48 -well plate, resulting in a $2 \%$ final concentration of fetal bovine serum and a MOI of 0.1. Plates were then centrifuged at 1,600 r.p.m. for $5 \mathrm{~min}$ before a $15-\mathrm{min}$ incubation at $37^{\circ} \mathrm{C}$. A total of $25 \mu \mathrm{l}$ was then collected from each well and serially diluted in a 96-well plate containing sterile water $(225 \mu \mathrm{l}$ per well). Samples $(25 \mu \mathrm{l})$ from each well were collected and plated on Todd Hewitt agar (THA) plates, which were incubated at $37^{\circ} \mathrm{C}$ overnight. Colonies were counted the next day to calculate bacterial survival. For bactericidal assays using opsonized bacteria, USA300 MRSA were incubated for $30 \mathrm{~min}$ in $80 \% 56^{\circ} \mathrm{C}$ heat-inactivated pooled human serum in RPMI before being combined with neutrophils in a 96-well plate (such that the final concentration of serum was $2 \%$ and the MOI was 1 ). Samples were collected, serially diluted in water and plated on THA plates to quantify CFUs.
Phagocytosis assay. Neutrophils were combined in a 96-well plate $\left(2 \times 10^{5}\right.$ cells per well) with pHrodo Red S. aureus Bioparticles (Life Technologies) as specified by the manufacturer. Plates were incubated at $37^{\circ} \mathrm{C}$, and phagocytosis was assessed by measuring fluorescence intensity $(560 \mathrm{~nm}$ excitation, $585 \mathrm{~nm}$ emission) at 15-min intervals using a SpectraMAX Gemini EM fluorescence reader (Molecular Devices).

Murine infection studies. For in vivo infection studies, 8-week-old female CD-1 mice (Charles River Laboratories, Wilmington, MA, USA) were injected intraperitoneally with tamoxifen $\left(250 \mathrm{mg} \mathrm{kg}^{-1}\right.$ ) or vehicle (corn oil) ( $n=6$ mice per group). After an hour, mice were infected intraperitoneally with a lethal dose $\left(\sim 1 \times 10^{9} \mathrm{CFU}\right)$ of MRSA strain USA300 (UAMS 1182). The mice received additional tamoxifen or vehicle treatments 1 and $8 \mathrm{~h}$ following infection, and survival was monitored over 7 days. Mice that appeared moribund were humanely killed. To determine CFUs in tissues of tamoxifen-treated and control animals, mice were treated with tamoxifen or vehicle $2 \mathrm{~h}$ before and $6 \mathrm{~h}$ after i.p. infection with EGFP-expressing USA300 MRSA. Mice were killed $8 \mathrm{~h}$ post infection. Peritoneal lavage and blood samples were collected along with the kidneys, livers and spleens of the infected animals. Following homogenization of organs using a MagNA Lyser instrument (Roche Diagnostics Corporation, Indianapolis, IN), samples were serially diluted in PBS and plated on THA plates to determine CFUs per gram of tissue. Two hundred-microlitre samples of the peritoneal lavages were fixed via addition of $4 \%$ paraformaldehyde and imaged using the Zeiss AxioObserver system described above. This in vivo infection study was performed in duplicate, and data from one representative experiment are shown. All studies involving animals were reviewed and approved by the University of California San Diego Animal Care and Use Committee.

Statistical analysis. All of the statistical analyses described in the figure legends were performed using Prism v6.0. Unless otherwise noted, $n$ values indicate the number of replicates performed in at least three independent experiments.

\section{References}

1. Waters, E. A., Cronin, K. A., Graubard, B. I., Han, P. K. \& Freedman, A. N. Prevalence of tamoxifen use for breast cancer chemoprevention among U.S. women. Cancer Epidemiol. Biomarkers Prev. 19, 443-446 (2010).

2. World Health Organization. WHO Model List of Essential Medicines: 18th List (World Health Organization, Geneva, 2013).

3. Komm, B. S. \& Mirkin, S. An overview of current and emerging SERMs. J. Steroid Biochem. Mol. Biol. 143, 207-222 (2014).

4. Paul, R. et al. Both the immunosuppressant SR31747 and the antiestrogen tamoxifen bind to an emopamil-insensitive site of mammalian Delta8-Delta7 sterol isomerase. J. Pharmacol. Exp. Ther. 285, 1296-1302 (1998).

5. Mandlekar, S. \& Kong, A. N. Mechanisms of tamoxifen-induced apoptosis. Apoptosis 6, 469-477 (2001).

6. Ligeiro de Oliveira, A. P., Oliveira-Filho, R. M., da Silva, Z. L., Borelli, P. \& Tavares de Lima, W. Regulation of allergic lung inflammation in rats: interaction between estradiol and corticosterone. Neuroimmunomodulation 11, 20-27 (2004)

7. Moreland, J. G., Davis, A. P., Bailey, G., Nauseef, W. M. \& Lamb, F. S. Anion channels, including $\mathrm{ClC}-3$, are required for normal neutrophil oxidative function, phagocytosis, and transendothelial migration. J. Biol. Chem. 281, 12277-12288 (2006).

8. Baumruker, T., Bornancin, F. \& Billich, A. The role of sphingosine and ceramide kinases in inflammatory responses. Immunol. Lett. 96, 175-185 (2005).

9. Brinkmann, V. \& Zychlinsky, A. Beneficial suicide: why neutrophils die to make NETs. Nat. Rev. Microbiol. 5, 577-582 (2007).

10. von Kockritz-Blickwede, M. \& Nizet, V. Innate immunity turned inside-out: antimicrobial defense by phagocyte extracellular traps. J. Mol. Med. 87, 775-783 (2009)

11. von Köckritz-Blickwede, M., Chow, O., Ghochani, M. \& Nizet, V. in: Methods in Microbiology (eds Dieter, K. \& Stefan, H. E. K.) (Academic Press, 2010).

12. Sun, J. et al. Antagonists selective for estrogen receptor alpha. Endocrinology 143, 941-947 (2002)

13. Compton, D. R. et al. Pyrazolo[1,5-a]pyrimidines: estrogen receptor ligands possessing estrogen receptor beta antagonist activity. J. Med. Chem. 47, 5872-5893 (2004).

14. Bologa, C. G. et al. Virtual and biomolecular screening converge on a selective agonist for GPR30. Nat. Chem. Biol. 2, 207-212 (2006).

15. Schmidt, J. M. et al. De novo design, synthesis, and evaluation of novel nonsteroidal phenanthrene ligands for the estrogen receptor. J. Med. Chem. 46, 1408-1418 (2003).

16. Thomas, P., Pang, Y., Filardo, E. J. \& Dong, J. Identity of an estrogen membrane receptor coupled to a $\mathrm{G}$ protein in human breast cancer cells. Endocrinology 146, 624-632 (2005).

17. Fuchs, T. A. et al. Novel cell death program leads to neutrophil extracellular traps. J. Cell Biol. 176, 231-241 (2007). 
18. Chow, O. A. et al. Statins enhance formation of phagocyte extracellular traps. Cell Host Microbe 8, 445-454 (2010).

19. Gschwendt, M. et al. Inhibition of protein kinase $C \mathrm{mu}$ by various inhibitors. Differentiation from protein kinase c isoenzymes. FEBS Lett. 392, 77-80 (1996).

20. Laudanna, C., Mochly-Rosen, D., Liron, T., Constantin, G. \& Butcher, E. C. Evidence of zeta protein kinase $\mathrm{C}$ involvement in polymorphonuclear neutrophil integrin-dependent adhesion and chemotaxis. J. Biol. Chem. 273, 30306-30315 (1998)

21. Lozano, J. et al. Protein kinase C zeta isoform is critical for kappa B-dependent promoter activation by sphingomyelinase. J. Biol. Chem. 269, 19200-19202 (1994).

22. Rani, C. S. et al. Cell cycle arrest induced by an inhibitor of glucosylceramide synthase. Correlation with cyclin-dependent kinases. J. Biol. Chem. 270, 2859-2867 (1995)

23. Saddoughi, S. A., Song, P. \& Ogretmen, B. Roles of bioactive sphingolipids in cancer biology and therapeutics. Subcell. Biochem. 49, 413-440 (2008).

24. Wadsworth, J. M. et al. The chemical basis of serine palmitoyltransferase inhibition by myriocin. J. Am. Chem. Soc. 135, 14276-14285 (2013).

25. Scarlatti, F. et al. Ceramide-mediated macroautophagy involves inhibition of protein kinase B and up-regulation of beclin 1. J. Biol. Chem. 279, 18384-18391 (2004).

26. Menegazzi, R., Decleva, E. \& Dri, P. Killing by neutrophil extracellular traps: fact or folklore? Blood 119, 1214-1216 (2012).

27. Malachowa, N., Kobayashi, S. D., Freedman, B., Dorward, D. W. \& DeLeo, F. R. Staphylococcus aureus leukotoxin GH promotes formation of neutrophil extracellular traps. J. Immunol. 191, 6022-6029 (2013).

28. Kobayashi, S. D. et al. Rapid neutrophil destruction following phagocytosis of Staphylococcus aureus. J. Innate Immun. 2, 560-575 (2010).

29. Seumois, G. et al. De novo C16- and C24-ceramide generation contributes to spontaneous neutrophil apoptosis. J. Leukoc. Biol. 81, 1477-1486 (2007).

30. Scheel-Toellner, D. et al. Clustering of death receptors in lipid rafts initiates neutrophil spontaneous apoptosis. Biochem. Soc. Trans. 32, 679-681 (2004).

31. Wartha, F. \& Henriques-Normark, B. ETosis: a novel cell death pathway. Sci. Signal. 1, pe25 (2008).

32. Maiuri, M. C., Zalckvar, E., Kimchi, A. \& Kroemer, G. Self-eating and self-killing: crosstalk between autophagy and apoptosis. Nat. Rev. Mol. Cell Biol. 8, 741-752 (2007)

33. Remijsen, Q. et al. Neutrophil extracellular trap cell death requires both autophagy and superoxide generation. Cell Res. 21, 290-304 (2011).

34. Uchiyama, S., Andreoni, F., Schuepbach, R. A., Nizet, V. \& Zinkernagel, A. S. DNase Sdal allows invasive M1T1 group A Streptococcus to prevent TLR9-dependent recognition. PLoS Pathog. 8, e1002736 (2012).

35. Schilcher, K. et al. Increased neutrophil extracellular trap-mediated Staphylococcus aureus clearance through inhibition of nuclease activity by clindamycin and immunoglobulin. J. Infect. Dis. 10, 473-482 (2014).
36. Ko, Y.-P. et al. Phagocytosis Escape by a Staphylococcus aureus protein that connects complement and coagulation proteins at the bacterial surface. PLoS Pathog. 9, e1003816 (2013).

37. Laarman, A. J. et al. Staphylococcus aureus metalloprotease aureolysin cleaves complement C3 to mediate immune evasion. J. Immunol. 186, 6445-6453 (2011).

38. Liu, G. Y. et al. Staphylococcus aureus golden pigment impairs neutrophil killing and promotes virulence through its antioxidant activity. J. Exp. Med. 202, 209-215 (2005).

39. Kessenbrock, K. et al. Netting neutrophils in autoimmune small-vessel vasculitis. Nat. Med. 15, 623-625 (2009).

40. Dworski, R., Simon, H. U., Hoskins, A. \& Yousefi, S. Eosinophil and neutrophil extracellular DNA traps in human allergic asthmatic airways. J. Allergy Clin. Immunol. 127, 1260-1266 (2011).

41. Zhang, R., Shen, Z., Nauseef, W. M. \& Hazen, S. L. Defects in leukocyte-mediated initiation of lipid peroxidation in plasma as studied in myeloperoxidase-deficient subjects: systematic identification of multiple endogenous diffusible substrates for myeloperoxidase in plasma. Blood 99, 1802-1810 (2002).

\section{Acknowledgements}

Research was supported by NIH grants HD071600, AI057153 and AI052453 (to V.N.) and OD008469 (to J.T.C.).

\section{Author contributions}

All authors planned and designed experiments. R.C., A.H., J.O., J.D., J.L. and D.J.G. conducted experiments. All authors interpreted data. R.C. and V.N. drafted and revised the manuscript.

\section{Additional information}

Supplementary Information accompanies this paper at http://www.nature.com/ naturecommunications

Competing financial interests: The authors declare no competing financial interests.

Reprints and permission information is available online at http://npg.nature.com/ reprintsandpermissions/

How to cite this article: Corriden, R. et al. Tamoxifen augments the innate immune function of neutrophils through modulation of intracellular ceramide. Nat. Commun. 6:8369 doi: 10.1038/ncomms9369 (2015). 